The Free Internet Journal

for Organic Chemistry
Paper

Arkivoc 2017, part iii, 279-286

\title{
Functionally substituted aromatic aldehydes as reagents in the synthesis of new substituted thioglycolurils
}

\author{
Galina A. Gazieva, ${ }^{* a}$ Sergei A. Serkov, ${ }^{a}$ Natalya V. Sigay, ${ }^{a}$ Natalya N. Kostikova, ${ }^{a}$ \\ Leonid D. Popov, ${ }^{b}$ and Angelina N. Kravchenko ${ }^{a}$
}

a N. D. Zelinsky Institute of Organic Chemistry, Russian Academy of Sciences, 47 Leninsky prosp., 119991 Moscow, Russia

${ }^{b}$ Department of Chemistry, Southern Federal University, 7 Zorge Street, 344090 Rostov-on-Don, Russia Email:gaz@ioc.ac.ru

Dedicated to Prof. Oleg A. Rakitin on the occasion of his 65th anniversary

Received 06-30-2017

Accepted 08-11-2017

Published on line $08-23-2017$

\section{Abstract}

Simple approach to the synthesis of 2-(4(2)-(((4,6-dialkyl-5-oxo-2-thioxohexahydroimidazo[4,5- $d$ ] imidazol$1(2 \mathrm{H})$-yl)imino)methyl)phenoxy)acetates or acetamides (new substituted thioglycolurils) based on the reaction of 5,7-dialkyl-3-thioxoperhydroimidazo[4,5-e]-1,2,4-triazine-6-ones with functionally substituted aromatic aldehydes has been developed. Synthesized thioglycolurils with acetate function can undergo further simple transformation to the corresponding acetamides.

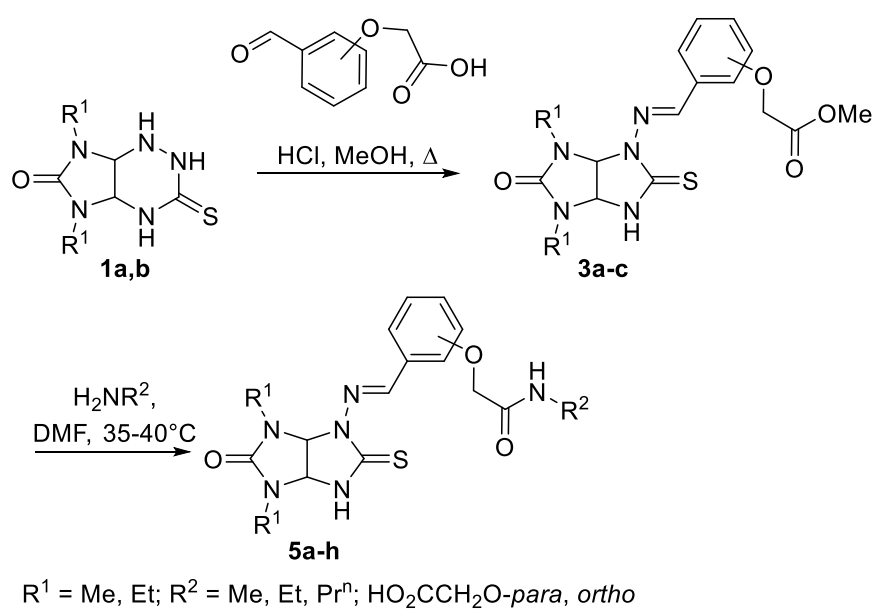

Keywords: Imidazotriazines, ring contraction, 4(2)-formylphenoxyacetic acids, thioglycolurils, primary amines 


\section{Introduction}

The progress in the chemistry of 5-thioxohexahydroimidazo[4,5-d]imidazol-2(1H)-ones (monothioanalogues of glycolurils) is of great interest due to various practically useful properties of both glycolurils ${ }^{1-4}$ and their iminoand thioanalogues. ${ }^{5-15}$ Thioanalogues of glycolurils have already been recognized as substrates for the template-directed crossed-Claisen condensation, ${ }^{8-11}$ building blocks for the synthesis of semithiobambusurils, ${ }^{12}$ organocatalysts for $\mathrm{N}$-Boc protection of amines ${ }^{13}$ or $\alpha$-monobromination of 1,3 dicarbonyl compounds, ${ }^{14}$ and as anxiolytic agents. ${ }^{15}$

There are different methods known for the synthesis of thioglycolurils, including recently reported approach based on the tandem hydrazone formation and triazine ring contraction of 5,7-dialkyl-3thioxoperhydroimidazo[4,5-e]-1,2,4-triazine-6-ones 1 with aromatic aldehydes (Scheme 1). ${ }^{16}$

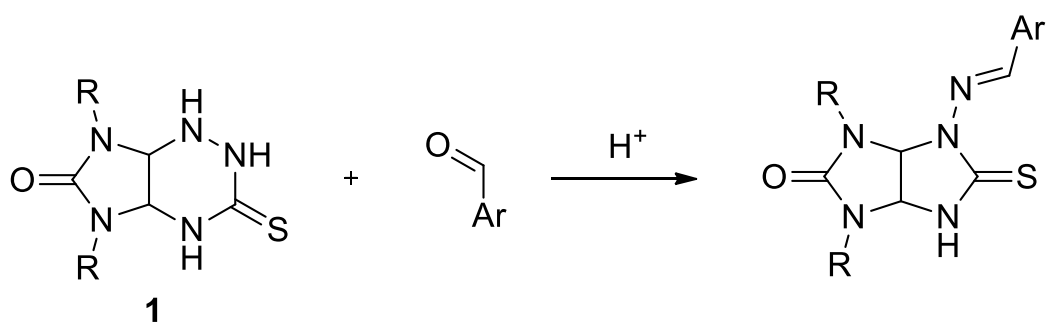

Scheme 1. Reaction of imidazotriazines 1 with aromatic aldehydes.

Some monothioanalogues of glycolurils prepared by this method demonstrated sedative ${ }^{17}$ or cytotoxic ${ }^{18}$ activities.

Substituted aromatic aldehydes bearing other functional groups could be used to introduce different additional moieties including pharmacophore ones in thioglycoluril framework.

In the present paper we have synthesized monothioanalogues of glycoluril by the reaction of 5,7-dialkyl-3thioxoperhydroimidazo[4,5-e]-1,2,4-triazine-6-ones 1 with aromatic aldehydes bearing substituent containing a carboxylic function and studied the reactivity of these thioglycolurils toward amines.

\section{Results and Discussion}

Recently we described a new method for the synthesis of substituted thioglycolurils by reaction of imidazotriazines 1 with (hetero)aromatic aldehydes which includes a tandem hydrazone formation and triazine ring contraction. ${ }^{19}$ Here we used this approach to the preparation of $\mathrm{N}$ (benzylideneamino)thioglycolurils which are functionally substituted in aromatic ring. Reaction of compounds $\mathbf{1} \mathbf{a}, \mathbf{b}$ and $4(2)$-formylphenoxyacetic acids $\mathbf{2 a}, \mathbf{b}$ in methanol in the presence of $\mathrm{HCl}$ led to methyl 2-(4(2)-(((4,6dialkyl-5-oxo-2-thioxohexahydroimidazo[4,5-d] imidazol-1(2H)-yl)imino)methyl)phenoxy)acetates 3a-c in 51$58 \%$ yield (Scheme 2). Apart from ring contraction reaction, esterification of carboxylic group with methanol occurred.

Reaction of methyl esters 3a-c with primary amines $4 a-c$ proceeded in DMF at room temperature and at $35-40{ }^{\circ} \mathrm{C}$ (Scheme 2). The reaction progress was monitored by recording ${ }^{1} \mathrm{H}$ NMR spectra of samples taken from the reaction mixture after $24,72,96$, and $120 \mathrm{~h}$. The signals of starting compounds 3a-c have 
disappeared at temperature $35-40{ }^{\circ} \mathrm{C}$ after $120 \mathrm{~h}$. The yields of corresponding amides $5 \mathrm{a}-\mathrm{h}$ were $52-93 \%$ (Table 1). At room temperature, conversion of starting compounds was not observed.

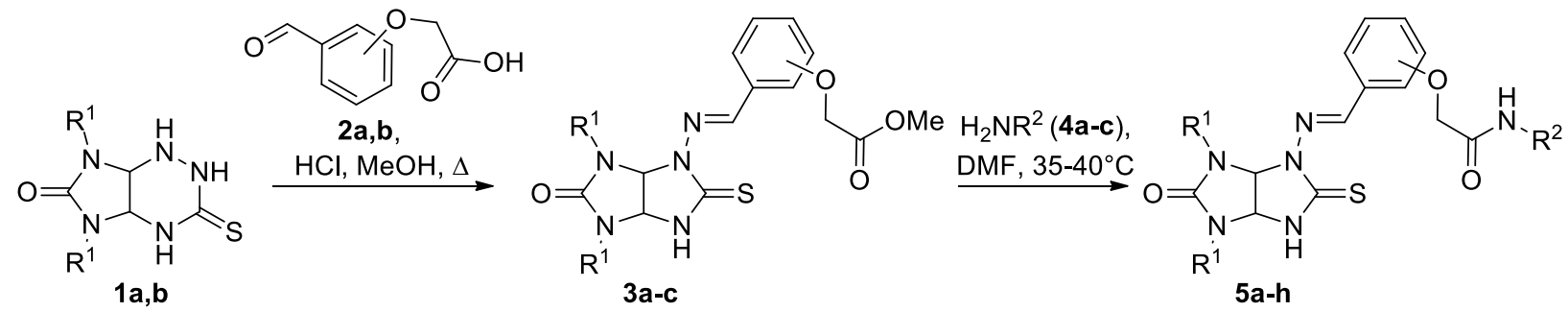

1a: $\mathrm{R}^{1}=$ Me; 1b: $\mathrm{R}^{1}=\mathrm{Et} ; \mathbf{2} \mathbf{a}$ : para; $\mathbf{2 b}$ : ortho; $\mathbf{3 a}: \mathrm{R}^{1}=$ Me, para; $\mathbf{3 b}: \mathrm{R}^{1}=\mathrm{Et}$, para; 3c: $\mathrm{R}^{1}=$ Me, ortho; 4a: $R^{2}=M e, 4 b: R^{2}=E t, 4 c: R^{2}=P^{n}$

Scheme 2. Synthesis of thioglycolurils 3a-c and their reaction with amines.

Table 1. Amides 5a-h prepared via reaction of esters 3a-c with primary amines $4 a-c$

\begin{tabular}{lllll}
\hline Amide 5 & $\mathrm{R}^{1}$ & $\mathrm{R}^{2}$ & Position of substituent & Yield (\%) $^{\mathrm{a}}$ \\
\hline $\mathbf{a}$ & $\mathrm{Me}$ & $\mathrm{Me}$ & para & 61 \\
$\mathbf{b}$ & $\mathrm{Me}$ & $\mathrm{Et}$ & para & 56 \\
$\mathbf{c}$ & $\mathrm{Me}$ & $\mathrm{Pr}^{\mathrm{n}}$ & para & 65 \\
$\mathbf{d}$ & $\mathrm{Et}$ & $\mathrm{Me}$ & para & 74 \\
$\mathbf{e}$ & $\mathrm{Et}$ & $\mathrm{Et}$ & para & 82 \\
$\mathbf{f}$ & $\mathrm{Et}$ & $\mathrm{Pr}^{\mathrm{n}}$ & para & 93 \\
$\mathbf{g}$ & $\mathrm{Me}$ & $\mathrm{Me}$ & ortho & 52 \\
$\mathbf{h}$ & $\mathrm{Me}$ & $\mathrm{Et}$ & ortho & 57 \\
\hline
\end{tabular}

ayield of isolated product.

We used another sequence of reactions to prepare amide $\mathbf{5 i}$ containing morpholine fragment since its direct synthesis usually requires harsh conditions ${ }^{20-22}$. Imidazotriazine $\mathbf{1 b}$ reacted with previously prepared ${ }^{23}$ 4-(2-morpholino-2-oxoethoxy)benzaldehyde 6 ( Scheme 3). Thioglycoluril 5i was synthesized in 53\% yield.<smiles>CCN1C(=O)N(CC)C2NC(=S)NNC21</smiles>

$1 \mathrm{~b}$

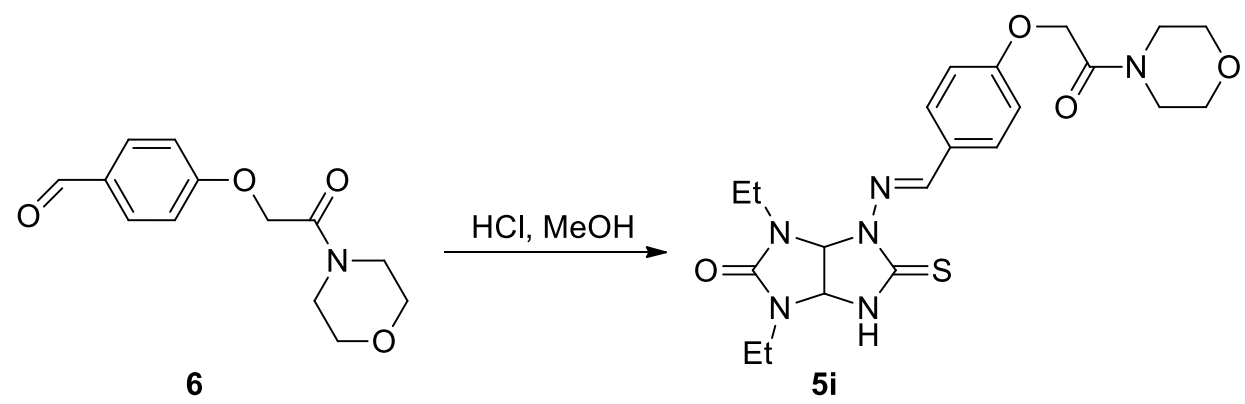

Scheme 3. Synthesis of amide $\mathbf{5 i}$.

The structures of thioglycoluryls 3 and 5 was ascertained by the IR, ${ }^{1} \mathrm{H} N M R,{ }^{13} \mathrm{C} N M R$, and HRMS spectral data. Earlier reported 1,3-dialkyl-4-(benzylideneamino)- and 4-((hetarylmethyliden)amino)thioglycolurils 
possessed only the E-configuration, which was confirmed both by NMR spectroscopy ${ }^{24}$ and by X-ray analysis data. ${ }^{16,18,19}$

\section{Conclusions}

A simple approach to the synthesis of new thioglycolurils with ester or amide function in the substituent based on reaction of 5,7-dialkyl-3-thioxoperhydroimidazo[4,5-e]-1,2,4-triazine-6-ones with functionally substituted aromatic aldehydes has been developed. This reaction comprises the tandem hydrazone formation and triazine ring contraction. An amide function can be previously introduced into molecule of benzaldehyde or obtained by the reaction of thioglycolurils bearing ester function in the substituent with primary amines. This approach allows to introduce different additional functionally groups including pharmacophore ones as morpholine for example.

\section{Experimental Section}

General. All reagents were purchased from Acros organics and used without further purification. Melting points were determined in open glass capillaries on a Gallenkamp (Sanyo) melting point apparatus. The ${ }^{1} \mathrm{H}$ $\mathrm{NMR}$ and ${ }^{13} \mathrm{C}$ NMR spectra were recorded on Bruker AM300 (300.13 MHz and $75.5 \mathrm{MHz}$, respectively) and Bruker DRX500 (500.13 MHz and $125.76 \mathrm{MHz}$, respectively) spectrometers using DMSO- $d_{6}$ as solvent. Chemical shifts $(\delta)$ are given in ppm from TMS as internal standard. Infrared (IR) spectra were recorded on a Bruker ALPHA instrument as $\mathrm{KBr}$ pellets. High resolution mass spectra (HRMS) were measured on a Bruker micrOTOF II instrument using electrospray ionization (ESI). 4(2)-Formylphenoxyacetic acids 2a,b and 4-(2morpholino-2-oxoethoxy)benzaldehyde (6) were prepared according to the known procedures. ${ }^{23,25,26}$

Methyl 2-(4(2)-((4,6-dialkyl-5-oxo-2-thioxohexahydroimidazo[4,5-d]imidazol-1(2H)-yl)imino)methyl)phenoxy)acetates (3a-c) and 1,3-diethyl-4-((4-(2-morpholino-2-oxoethoxy)benzylidene)amino)-5thioxohexahydroimidazo[4,5-d]imidazol-2(1H)-one (5i). To a stirred suspension of 5,7-dialkyl-3thioxoperhydroimidazo[4,5-e]-1,2,4-triazin-6-one $1 \mathbf{a}$ or $\mathbf{1 b}(2 \mathrm{mmol})$ in methanol (30 $\mathrm{mL}$ ), two drops of concentrated $\mathrm{HCl}$ and an aldehyde $\mathbf{2 a}$ or $\mathbf{2 b}$ or $\mathbf{6}(2 \mathrm{mmol})$ were added. The resulting mixture was heated to reflux and stirred for $1.5 \mathrm{~h}(2 \mathrm{~h}$ for $5 \mathrm{i}$ ), then concentrated to dryness. The residue was recrystallized from EtOH (3a-c) or $\mathrm{MeOH}-\mathrm{H}_{2} \mathrm{O}(10: 2)(5 \mathbf{i})$ to give desired thioglycoluril 3 or $\mathbf{5 i}$ :

Methyl 2-(4-((4,6-dimethyl-5-oxo-2-thioxohexahydroimidazo[4,5-d]imidazol-1(2H)-yl)imino)methyl)phenoxy)acetate (3a). White solid (0.383 g, 51\%), mp 169-171 ${ }^{\circ} \mathrm{C} . \mathrm{IR}(\mathrm{KBr}) \mathrm{v}, \mathrm{cm}^{-1}: 3207,1766,1721,1611$, 1598, 1509, 1412, 1298, 1269, 1254, 1212, 1177, 1071, 1055, 1032. ${ }^{1} \mathrm{H}$ NMR (300 MHz, DMSO-d $\left.d_{6}\right): \delta 2.75$ (s, $3 \mathrm{H}, \mathrm{NMe}), 2.84(\mathrm{~s}, 3 \mathrm{H}, \mathrm{NMe}), 3.71(\mathrm{~s}, 3 \mathrm{H}, \mathrm{OMe}), 4.88\left(\mathrm{~s}, 2 \mathrm{H}, \mathrm{OCH}_{2}\right), 5.38(\mathrm{~d}, 3 \mathrm{~J} 8.2 \mathrm{~Hz}, 1 \mathrm{H}, \mathrm{CH}), 5.91\left(\mathrm{~d}, 3^{3} 8.2 \mathrm{~Hz}\right.$, $1 \mathrm{H}, \mathrm{CH}), 7.04\left(\mathrm{~d},{ }^{3} \mathrm{~J} 8.6 \mathrm{~Hz}, 2 \mathrm{H}, \mathrm{CH}_{\text {arom }}\right), 7.71$ (d, $\left.{ }^{3} \mathrm{~J} 8.6 \mathrm{~Hz}, 2 \mathrm{H}, \mathrm{CH}_{\text {arom }}\right), 9.04(\mathrm{~s}, 1 \mathrm{H}, \mathrm{N}=\mathrm{CH}), 9.91(\mathrm{~s}, 1 \mathrm{H}, \mathrm{NH}) .{ }^{13} \mathrm{C}$ NMR (75 MHz, DMSO-d $)$ : $\delta$ 28.2, 30.0 (NMe), $51.8(\mathrm{OMe}), 64.6\left(\mathrm{OCH}_{2}\right), 68.0,75.3(\mathrm{CH}), 114.9\left(2 \mathrm{CH}_{\mathrm{arom}}\right), 127.0$ (C $\left.\mathrm{C}_{\text {arom }}\right), 129.0\left(2 \mathrm{CH}_{\text {arom }}\right), 152.7(\mathrm{~N}=\mathrm{CH}), 157.5(\mathrm{C}=\mathrm{O}), 159.6\left(\mathrm{C}_{\text {arom }}\right), 168.9(\mathrm{C}=\mathrm{O}), 178.9$ ( $\left.\mathrm{C}=\mathrm{S}\right) . \mathrm{HRMS}(\mathrm{ESI}): \mathrm{m} / \mathrm{z}$ calcd for $\mathrm{C}_{16} \mathrm{H}_{19} \mathrm{~N}_{5} \mathrm{O}_{4} \mathrm{~S}+\mathrm{Na}^{+}$: 400.1050; found: 400.1039;

Methyl 2-(4-(((4,6-diethyl-5-oxo-2-thioxohexahydroimidazo[4,5-d]imidazol-1(2H)-yl)imino)methyl)phenoxy)acetate (3b). White solid (0.47 g, 58\%), mp 207-209 ${ }^{\circ} \mathrm{C}$. IR (KBr) v, cm ${ }^{-1}: 3180,1737,1704,1611,1595,1508$, $1474,1429,1311,1275,1248,1207,1166,1067 .{ }^{1} \mathrm{H}$ NMR $\left(300 \mathrm{MHz}, \mathrm{DMSO}-d_{6}\right): \delta 1.01-1.10$ (m, 6H, Me), $3.11-$ 
$3.38\left(\mathrm{~m}, 4 \mathrm{H}, \mathrm{NCH}_{2}\right), 3.71(\mathrm{~s}, 3 \mathrm{H}, \mathrm{OMe}), 4.88\left(\mathrm{~s}, 2 \mathrm{H}, \mathrm{OCH}_{2}\right), 5.49$ (d, $\left.{ }^{3} \mathrm{~J} 8.4 \mathrm{~Hz}, 1 \mathrm{H}, \mathrm{CH}\right), 5.94\left(\mathrm{~d}, 3^{3} \mathrm{~J} .4 \mathrm{~Hz}, 1 \mathrm{H}, \mathrm{CH}\right)$, 7.04 (d, ${ }^{3}$ s $\left.8.6 \mathrm{~Hz}, 2 \mathrm{H}, \mathrm{CH}_{\text {arom }}\right), 7.70$ (d, $\left.{ }^{3} \mathrm{~J} 8.6 \mathrm{~Hz}, 2 \mathrm{H}, \mathrm{CH}_{\text {arom }}\right), 9.15$ (s, $\left.1 \mathrm{H}, \mathrm{N}=\mathrm{CH}\right), 9.88$ (s, 1H, NH) . ${ }^{13} \mathrm{C} \mathrm{NMR}(75$ $\left.\mathrm{MHz}, \mathrm{DMSO}-d_{6}\right): \delta$ 12.9, $13.4(\mathrm{Me}), 35.9,37.0\left(\mathrm{NCH}_{2}\right), 51.8(\mathrm{OMe}), 64.6\left(\mathrm{OCH}_{2}\right), 66.1,74.9(\mathrm{CH}), 115.0$ $\left(2 \mathrm{CH}_{\text {arom }}\right), 126.8$ ( $\left.\mathrm{C}_{\text {arom }}\right), 129.1\left(2 \mathrm{CH}_{\text {arom }}\right), 154.9(\mathrm{~N}=\mathrm{CH}), 156.8(\mathrm{C}=\mathrm{O}), 159.7$ ( $\left.\mathrm{C}_{\text {arom }}\right), 168.9(\mathrm{C}=0), 178.6(\mathrm{C}=\mathrm{S})$. HRMS (ESI): $m / z$ calcd for $\mathrm{C}_{18} \mathrm{H}_{23} \mathrm{~N}_{5} \mathrm{O}_{4} \mathrm{~S}+\mathrm{Na}^{+}:$: 428.1363; found: 428.1352 .

Methyl 2-(2-((4,6-dimethyl-5-oxo-2-thioxohexahydroimidazo[4,5-d]imidazol-1(2H)-yl)imino)methyl)phenoxy)acetate (3c). Pinkish solid (0.415 g, 55\%), mp 178-179 ${ }^{\circ} \mathrm{C}$. IR (KBr) v, cm ${ }^{-1}: 3180,1764,1720,1602$, 1523, 1492, 1449, 1420, 1272, 1213, 1161, 1115, 1075, 1058, 1043, 748. ${ }^{1} \mathrm{H}$ NMR (500 MHz, DMSO-d $): \delta 2.76$ (s, 3H, NMe), 2.90 (s, 3H, NMe), 3.71 (s, 3H, OMe), 4.90 (d, 2J $16.7 \mathrm{~Hz}, 1 \mathrm{H}, \mathrm{OCH}_{2}$ ), 4.94 (d, 2J $16.7 \mathrm{~Hz}, 1 \mathrm{H}, \mathrm{OCH}_{2}$ ),

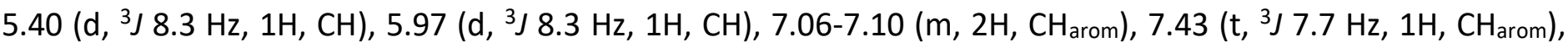
7.87 (d, ${ }^{3}$ 7.7 Hz, $\left.1 \mathrm{H}, \mathrm{CH}_{\text {arom }}\right), 9.35$ (s, $\left.1 \mathrm{H}, \mathrm{N}=\mathrm{CH}\right), 10.02(\mathrm{~s}, 1 \mathrm{H}, \mathrm{NH}) .{ }^{13} \mathrm{C} \mathrm{NMR}\left(75 \mathrm{MHz}, \mathrm{DMSO}-d_{6}\right): \delta 28.2,30.6$ (NMe), 51.9 (OMe), $65.2\left(\mathrm{OCH}_{2}\right), 68.1,75.1(\mathrm{CH}), 113.1,121.6\left(\mathrm{CH}_{\text {arom }}\right), 122.5\left(\mathrm{C}_{\text {arom }}\right), 125.8,131.9\left(\mathrm{CH}_{\text {arom }}\right)$, $145.7(\mathrm{~N}=\mathrm{CH}), 156.5,157.7\left(\mathrm{C}_{\text {arom }}, \mathrm{C}=\mathrm{O}\right), 168.9(\mathrm{C}=\mathrm{O}), 179.0(\mathrm{C}=\mathrm{S})$. HRMS (ESI): $\mathrm{m} / \mathrm{z}$ calcd for $\mathrm{C}_{16} \mathrm{H}_{19} \mathrm{~N}_{5} \mathrm{O}_{4} \mathrm{~S}+\mathrm{Na}^{+}$: 400.1050; found: 400.1041.

\section{1,3-Diethyl-4-((4-(2-morpholino-2-oxoethoxy)benzylidene)amino)-5-thioxohexahydroimidazo[4,5-d]-}

imidazol-2(1H)-one (5i). White solid (0.488 g, 53\%), mp 203-205 ${ }^{\circ} \mathrm{C} . \mathrm{IR}(\mathrm{KBr}) \mathrm{v}, \mathrm{cm}^{-1}: 3261,1701,1678,1605$, $1512,1503,1474,1453,1436,1311,1276,1255,1233,1200,1172,1107,1074,1055,1033,795 .{ }^{1} \mathrm{H}$ NMR (300 $\left.\mathrm{MHz}, \mathrm{DMSO}-d_{6}\right): \delta$ 1.03-1.12 (m, 6H, Me), 3.12-3.37 (m, 4H, NCH$), 3.46-3.37\left(\mathrm{~m}, 8 \mathrm{H}, \mathrm{CH}_{2 m o r p h}\right), 4.95(\mathrm{~s}, 2 \mathrm{H}$,

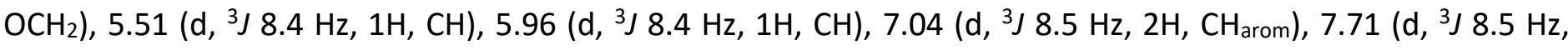
$\left.2 \mathrm{H}, \mathrm{CH}_{\text {arom }}\right), 9.16(\mathrm{~s}, 1 \mathrm{H}, \mathrm{N}=\mathrm{CH}), 9.90(\mathrm{~s}, 1 \mathrm{H}, \mathrm{NH}) .{ }^{13} \mathrm{C} \mathrm{NMR}\left(125 \mathrm{MHz}, \mathrm{DMSO}-d_{6}\right): \delta$ 12.9, $13.4(\mathrm{Me}), 35.9,37.0$ $\left(\mathrm{NCH}_{2}\right), 41.6,44.6\left(\mathrm{NCH}_{2 \mathrm{morph}}\right), 65.7\left(\mathrm{OCH}_{2}\right), 65.99(\mathrm{CH}), 66.05,66.1\left(\mathrm{OCH}_{2 \mathrm{morph}}\right), 75.0(\mathrm{CH}), 115.1\left(\mathrm{CH}_{\mathrm{arom}}\right), 126.4$ (C $\left.\mathrm{C}_{\text {arom}}\right), 129.0\left(\mathrm{CH}_{\text {arom }}\right), 155.3(\mathrm{~N}=\mathrm{CH}), 156.8(\mathrm{C}=\mathrm{O}), 160.3\left(\mathrm{C}_{\text {arom }}\right), 165.7(\mathrm{C}=\mathrm{O}), 178.6(\mathrm{C}=\mathrm{S}) . \mathrm{HRMS}(\mathrm{ESI}): \mathrm{m} / \mathrm{z}$ calcd for $\mathrm{C}_{21} \mathrm{H}_{28} \mathrm{~N}_{6} \mathrm{O}_{4} \mathrm{~S}+\mathrm{H}^{+}: 461.1966$; found: 461.1964 .

\section{2-(4(2)-(((4,6-Dialkyl-5-oxo-2-thioxohexahydroimidazo[4,5-d]imidazol-1(2H)-yl)imino)methyl)phenoxy)- $\mathrm{N}$ -}

alkylacetamides (5a-h). To a stirred solution of ester $\mathbf{3 a}$ or $\mathbf{3 b}$ or $\mathbf{3 c}(1 \mathrm{mmol})$ in DMF $(7 \mathrm{~mL})$, corresponding primary amine $\mathbf{4 a}$ or $\mathbf{4 b}$ or $\mathbf{4 c}(5 \mathrm{mmol})$ was added. The resulting mixture was stirred at temperature $35-40{ }^{\circ} \mathrm{C}$ for 5 days, then concentrated to dryness and diluted with water. A precipitate was recrystallized from EtOH or $\mathrm{MeOH}$ to give desired amide 5.

\section{2-(4-(((4,6-Dimethyl-5-oxo-2-thioxohexahydroimidazo[4,5-d]imidazol-1(2H)-yl)imino)methyl)phenoxy)- $N$ -}

methylacetamide (5a). White solid (0.229 g, 61\%), mp 233-235 ${ }^{\circ} \mathrm{C} . \mathrm{IR}(\mathrm{KBr}) \mathrm{v}, \mathrm{cm}^{-1}$ : 3390, 3289, 1696, 1661, $1608,1546,1511,1418,1404,1252,1208,1174,1057,1047 .{ }^{1} \mathrm{H}$ NMR (300 MHz, DMSO-d 6 ): $\delta 2.66$ (br.s, $3 \mathrm{H}$, $\mathrm{NMe}), 2.75$ (s, 3H, NMe), $2.84(\mathrm{~s}, 3 \mathrm{H}, \mathrm{NMe}), 4.53\left(\mathrm{~s}, 2 \mathrm{H}, \mathrm{OCH}_{2}\right), 5.38(\mathrm{~d}, 3 \mathrm{~J} 8.0 \mathrm{~Hz}, 1 \mathrm{H}, \mathrm{CH}), 5.92(\mathrm{~d}, 3 \mathrm{~J} 7.9 \mathrm{~Hz}, 1 \mathrm{H}$, $\mathrm{CH}$ ), 7.06 (d, 3 $7.6 \mathrm{~Hz}, 2 \mathrm{H}, \mathrm{CH}_{\text {arom }}$ ), 7.72 (d, 3J $\left.7.6 \mathrm{~Hz}, 2 \mathrm{H}, \mathrm{CH}_{\text {arom }}\right), 8.07$ (br.s, $1 \mathrm{H}, \mathrm{NH}$ ), 9.03 (s, $1 \mathrm{H}, \mathrm{N}=\mathrm{CH}$ ), 9.92 (s, $1 \mathrm{H}, \mathrm{NH}) .{ }^{13} \mathrm{C}$ NMR $\left(75 \mathrm{MHz}, \mathrm{DMSO}-d_{6}\right): \delta 25.3(\mathrm{NMe}), 28.1,29.9(\mathrm{NMe}), 67.0(\mathrm{CH}), 68.0\left(\mathrm{OCH}_{2}\right), 75.3(\mathrm{CH}), 115.1$ $\left(2 \mathrm{CH}_{\text {arom }}\right), 126.9$ ( $\left.\mathrm{C}_{\text {arom }}\right), 129.0\left(2 \mathrm{CH}_{\text {arom }}\right), 152.8(\mathrm{~N}=\mathrm{CH}), 157.5(\mathrm{C}=\mathrm{O}), 159.7$ ( $\left.\mathrm{C}_{\text {arom }}\right), 167.6(\mathrm{C}=\mathrm{O}), 178.8(\mathrm{C}=\mathrm{S})$. HRMS (ESI): $m / z$ calcd for $\mathrm{C}_{16} \mathrm{H}_{20} \mathrm{~N}_{6} \mathrm{O}_{3} \mathrm{~S}+\mathrm{Na}^{+}$: 399.1210; found: 399.1204 .

2-(4-((4,6-Dimethyl-5-oxo-2-thioxohexahydroimidazo[4,5-d]imidazol-1(2H)-yl)imino)methyl)phenoxy)-Nethylacetamide (5b). White solid (0.219 g, 56\%), mp 121-123 ${ }^{\circ} \mathrm{C} . \mathrm{IR}(\mathrm{KBr}) \mathrm{v}, \mathrm{cm}^{-1}: 3457,3280,1692,1655$, $1609,1552,1510,1445,1416,1252,1212,1174,1050,793 .{ }^{1} \mathrm{H}$ NMR (300 MHz, DMSO-d 6 ): $\delta 1.04\left(\mathrm{t},{ }^{3} \mathrm{~J} 6.9 \mathrm{~Hz}\right.$, $3 \mathrm{H}, \mathrm{Me}), 2.75$ (s, 3H, NMe), $2.84(\mathrm{~s}, 3 \mathrm{H}, \mathrm{NMe}), 3.12-3.19\left(\mathrm{~m}, 2 \mathrm{H}, \mathrm{NCH}_{2}\right), 4.52\left(\mathrm{~s}, 2 \mathrm{H}, \mathrm{OCH}_{2}\right), 5.38(\mathrm{~d}, 3 \mathrm{~J} 8.0 \mathrm{~Hz}$, $1 \mathrm{H}, \mathrm{CH}), 5.91(\mathrm{~d}, 3 \mathrm{~J} 8.0 \mathrm{~Hz}, 1 \mathrm{H}, \mathrm{CH}), 7.06\left(\mathrm{~d}, 3 \mathrm{~J} 8.0 \mathrm{~Hz}, 2 \mathrm{H}, \mathrm{CH}_{\text {arom }}\right), 7.72$ (d, 3J $\left.8.0 \mathrm{~Hz}, 2 \mathrm{H}, \mathrm{CH}_{\text {arom }}\right), 8.13$ (br.s, $1 \mathrm{H}$, $\mathrm{NH}), 9.03(\mathrm{~s}, 1 \mathrm{H}, \mathrm{N}=\mathrm{CH}), 9.91(\mathrm{~s}, 1 \mathrm{H}, \mathrm{NH}) .{ }^{13} \mathrm{C} \mathrm{NMR}\left(75 \mathrm{MHz}, \mathrm{DMSO}-d_{6}\right): \delta 14.7$ (Me), 28.1, 29.9 (NMe), 33.2 $\left(\mathrm{NCH}_{2}\right), 67.0(\mathrm{CH}), 68.0\left(\mathrm{OCH}_{2}\right), 75.3(\mathrm{CH}), 115.1\left(2 \mathrm{CH}_{\text {arom }}\right), 126.9\left(\mathrm{C}_{\text {arom }}\right), 129.0\left(2 \mathrm{CH}_{\text {arom }}\right), 152.9(\mathrm{~N}=\mathrm{CH}), 157.5$ 
$(\mathrm{C}=\mathrm{O}), 159.8\left(\mathrm{C}_{\text {arom }}\right), 166.9(\mathrm{C}=\mathrm{O}), 178.8(\mathrm{C}=\mathrm{S})$. HRMS (ESI): $\mathrm{m} / \mathrm{z}$ calcd for $\mathrm{C}_{17} \mathrm{H}_{22} \mathrm{~N}_{6} \mathrm{O}_{3} \mathrm{~S}+\mathrm{Na}^{+}$: 413.1366; found: 413.1370.

2-(4-((4,6-Dimethyl-5-oxo-2-thioxohexahydroimidazo[4,5-d]imidazol-1(2H)-yl)imino)methyl)phenoxy)- $N$ propylacetamide (5c). White solid $(0.263 \mathrm{~g}, 65 \%), \mathrm{mp} \mathrm{211-214}{ }^{\circ} \mathrm{C}$. IR (KBr) v, cm${ }^{-1}: 3399,3269,1690,1660$, $1604,1542,1512,1486,1458,1419,1250,1230,1204,1170,1040 .{ }^{1} \mathrm{H}$ NMR $(500 \mathrm{MHz}$, DMSO-d $): \delta 0.84\left(\mathrm{t},{ }^{3} \mathrm{~J}\right.$ $7.3 \mathrm{~Hz}, 3 \mathrm{H}, \mathrm{Me}), 1.43-1.47\left(\mathrm{~m}, 2 \mathrm{H}, \mathrm{CH}_{2}\right), 2.75$ (s, 3H, NMe), $2.84(\mathrm{~s}, 3 \mathrm{H}, \mathrm{NMe}), 3.08-3.11\left(\mathrm{~m}, 2 \mathrm{H}, \mathrm{NCH}_{2}\right), 4.54(\mathrm{~s}$, $2 \mathrm{H}, \mathrm{OCH}_{2}$ ), 5.39 (d, $\left.{ }^{3} \mathrm{~J} 8.1 \mathrm{~Hz}, 1 \mathrm{H}, \mathrm{CH}\right), 5.91\left(\mathrm{~d},{ }^{3} \mathrm{~J} 8.1 \mathrm{~Hz}, 1 \mathrm{H}, \mathrm{CH}\right), 7.06\left(\mathrm{~d}, 3 \mathrm{~J} 8.2 \mathrm{~Hz}, 2 \mathrm{H}, \mathrm{CH}_{\text {arom }}\right), 7.72\left(\mathrm{~d}, 3^{3} 8.2\right.$ $\left.\mathrm{Hz}, 2 \mathrm{H}, \mathrm{CH}_{\text {arom }}\right), 8.09$ (br.s, $\left.1 \mathrm{H}, \mathrm{NH}\right), 9.05(\mathrm{~s}, 1 \mathrm{H}, \mathrm{N}=\mathrm{CH}), 9.90(\mathrm{~s}, 1 \mathrm{H}, \mathrm{NH}) .{ }^{13} \mathrm{C} \mathrm{NMR}\left(75 \mathrm{MHz}, \mathrm{DMSO}-d_{6}\right): \delta 11.3$ (Me), $22.3\left(\mathrm{CH}_{2}\right), 28.1,30.0(\mathrm{NMe}), 40.1\left(\mathrm{NCH}_{2}\right), 67.0(\mathrm{CH}), 68.0\left(\mathrm{OCH}_{2}\right), 75.2(\mathrm{CH}), 115.1\left(2 \mathrm{CH}_{\text {arom }}\right), 126.9$ ( $\left.\mathrm{C}_{\text {arom }}\right), 129.0\left(2 \mathrm{CH}_{\text {arom }}\right), 152.7(\mathrm{~N}=\mathrm{CH}), 157.5(\mathrm{C}=\mathrm{O}), 159.8\left(\mathrm{C}_{\text {arom }}\right), 167.0(\mathrm{C}=\mathrm{O}), 178.9(\mathrm{C}=\mathrm{S}) . \mathrm{HRMS}(\mathrm{ESI}): \mathrm{m} / \mathrm{z}$ calcd for $\mathrm{C}_{18} \mathrm{H}_{24} \mathrm{~N}_{6} \mathrm{O}_{3} \mathrm{~S}+\mathrm{H}^{+}$: 405.1703; found: 405.1702 .

2-(4-((4,6-Diethyl-5-oxo-2-thioxohexahydroimidazo[4,5-d]imidazol-1(2H)-yl)imino)methyl)phenoxy)- $\mathrm{N}$ -

methylacetamide (5d). White solid (0.299 g, 74\%), mp 211-213 ${ }^{\circ} \mathrm{C}$. IR (KBr) v, cm${ }^{-1}: 3453,3177,1708,1686$, $16089,1594,1506,1474,1457,1428,1416,1246,1206,1165,1073,1045 .{ }^{1} \mathrm{H}$ NMR (300 MHz, DMSO-d $)^{2}: \delta$ 1.02-1.08 (m, 6H, Me), 2.66 (br.s, 3H, NMe), 3.08-3.34 (m, 4H, NCH 2$), 4.54\left(\mathrm{~s}, 2 \mathrm{H}, \mathrm{OCH}_{2}\right), 5.49(\mathrm{~d}, 3 \mathrm{~J} 8.0 \mathrm{~Hz}, 1 \mathrm{H}$,

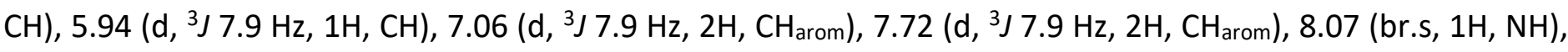
$9.14(\mathrm{~s}, 1 \mathrm{H}, \mathrm{N}=\mathrm{CH}), 9.88$ (s, $1 \mathrm{H}, \mathrm{NH}) .{ }^{13} \mathrm{C} \mathrm{NMR}\left(125 \mathrm{MHz}, \mathrm{DMSO}-d_{6}\right): \delta$ 12.8, 13.4 (Me), 25.3 (NMe), $35.9,37.0$ $\left(\mathrm{NCH}_{2}\right), 66.1(\mathrm{CH}), 67.0\left(\mathrm{OCH}_{2}\right), 74.9(\mathrm{CH}), 115.2\left(2 \mathrm{CH}_{\text {arom }}\right), 126.7\left(\mathrm{C}_{\text {arom }}\right), 129.1\left(2 \mathrm{CH}_{\text {arom }}\right), 155.0(\mathrm{~N}=\mathrm{CH}), 156.8$ $(\mathrm{C}=\mathrm{O}), 159.9\left(\mathrm{C}_{\text {arom }}\right), 167.6(\mathrm{C}=\mathrm{O}), 178.6(\mathrm{C}=\mathrm{S})$. HRMS (ESI): $\mathrm{m} / \mathrm{z}$ calcd for $\mathrm{C}_{18} \mathrm{H}_{24} \mathrm{~N}_{6} \mathrm{O}_{3} \mathrm{~S}+\mathrm{Na}^{+}$: 427.1523; found: 427.1519.

2-(4-(((4,6-Diethyl-5-oxo-2-thioxohexahydroimidazo[4,5-d]imidazol-1(2H)-yl)imino)methyl)phenoxy)- $\mathrm{N}$ ethylacetamide (5e). White solid (0.343 g, 82\%), mp 222-224 ${ }^{\circ} \mathrm{C}$ (decomp). IR (KBr) v, cm ${ }^{-1}: 3446,3165,1709$, $1683,1609,1594,1534,1507,1472,1451,1428,1279,1243,1206,1165,1073,1046 .{ }^{1} \mathrm{H} \mathrm{NMR}(300 \mathrm{MHz}$, DMSO- $\left.d_{6}\right): \delta$ 1.03-1.12 (m, 9H, Me), 3.14-3.24 (m, 4H, NCH$), 3.25-3.33\left(\mathrm{~m}, 2 \mathrm{H}, \mathrm{NCH}_{2}\right), 4.53\left(\mathrm{~s}, 2 \mathrm{H}, \mathrm{OCH}_{2}\right), 5.49$ (d, 3J $8.2 \mathrm{~Hz}, 1 \mathrm{H}, \mathrm{CH}$ ), 5.94 (d, 3J $8.1 \mathrm{~Hz}, 1 \mathrm{H}, \mathrm{CH}), 7.06$ (d, 3J $\left.8.4 \mathrm{~Hz}, 2 \mathrm{H}, \mathrm{CH}_{\text {arom }}\right), 7.72$ (d, 3J $8.4 \mathrm{~Hz}, 2 \mathrm{H}, \mathrm{CH}_{\text {arom }}$ ), 8.14 (br.s, $1 \mathrm{H}, \mathrm{NH}), 9.14$ (s, $1 \mathrm{H}, \mathrm{N}=\mathrm{CH}), 9.89$ (s, $1 \mathrm{H}, \mathrm{NH}) .{ }^{13} \mathrm{C} \mathrm{NMR}$ (75 MHz, DMSO-d $)$ ): $\delta$ 12.8, 13.4, 14.7 (Me), 33.2, 35.9, $37.0\left(\mathrm{NCH}_{2}\right), 66.1(\mathrm{CH}), 67.0\left(\mathrm{OCH}_{2}\right), 74.9(\mathrm{CH}), 115.2\left(2 \mathrm{CH}_{\text {arom }}\right), 126.7\left(\mathrm{C}_{\text {arom }}\right), 129.0\left(2 \mathrm{CH}_{\text {arom }}\right), 154.9$ $(\mathrm{N}=\mathrm{CH}), 156.8(\mathrm{C}=\mathrm{O}), 159.9\left(\mathrm{C}_{\text {arom }}\right), 166.9(\mathrm{C}=\mathrm{O}), 178.6(\mathrm{C}=\mathrm{S})$. HRMS (ESI): $\mathrm{m} / z$ calcd for $\mathrm{C}_{19} \mathrm{H}_{26} \mathrm{~N}_{6} \mathrm{O}_{3} \mathrm{~S}+\mathrm{H}^{+}$: 419.1860; found: 418.1849 .

2-(4-((4,6-Diethyl-5-oxo-2-thioxohexahydroimidazo[4,5- $d$ ]imidazol-1(2H)-yl)imino)methyl)phenoxy)- $\mathrm{N}$ propylacetamide (5f). White solid (0.402 g, 93\%), mp 190-192 ${ }^{\circ} \mathrm{C}$. IR (KBr) v, cm${ }^{-1}: 3434,3179,1709,1687$, $1595,1539,1507,1471,1444,1428,1277,1245,1206,1166,1072,1048 .{ }^{1} \mathrm{H}$ NMR (300 MHz, DMSO-d $\left.d_{6}\right): \delta 0.83$ (t, 3 J $7.1 \mathrm{~Hz}, 3 \mathrm{H}, \mathrm{Me}), 1.01-1.10(\mathrm{~m}, 6 \mathrm{H}, \mathrm{Me}), 1.41-1.48\left(\mathrm{~m}, 2 \mathrm{H}, \mathrm{CH}_{2}\right), 3.08-3.31\left(\mathrm{~m}, 6 \mathrm{H}, \mathrm{NCH}_{2}\right), 4.54(\mathrm{~s}, 2 \mathrm{H}$,

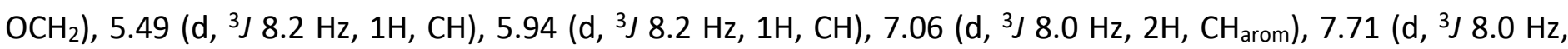
$\left.2 \mathrm{H}, \mathrm{CH}_{\text {arom }}\right), 8.11$ (br.s, $\left.1 \mathrm{H}, \mathrm{NH}\right), 9.14(\mathrm{~s}, 1 \mathrm{H}, \mathrm{N}=\mathrm{CH}), 9.88(\mathrm{~s}, 1 \mathrm{H}, \mathrm{NH}) .{ }^{13} \mathrm{C}$ NMR $\left(75 \mathrm{MHz}, \mathrm{DMSO}-d_{6}\right): \delta 11.2,12.8$, $13.3(\mathrm{Me}), 22.3\left(\mathrm{CH}_{2}\right), 35.9,37.0,40.0\left(\mathrm{NCH}_{2}\right), 66.1,67.0\left(\mathrm{CH}, \mathrm{OCH}_{2}\right), 74.9(\mathrm{CH}), 115.2\left(2 \mathrm{CH}_{\text {arom }}\right), 126.7\left(\mathrm{C}_{\text {arom }}\right)$, $129.0\left(2 \mathrm{CH}_{\text {arom }}\right), 154.9(\mathrm{~N}=\mathrm{CH}), 156.8(\mathrm{C}=\mathrm{O}), 159.9\left(\mathrm{C}_{\text {arom }}\right), 167.0(\mathrm{C}=\mathrm{O}), 178.6(\mathrm{C}=\mathrm{S})$. HRMS (ESI): $\mathrm{m} / \mathrm{z}$ calcd for $\mathrm{C}_{20} \mathrm{H}_{28} \mathrm{~N}_{6} \mathrm{O}_{3} \mathrm{~S}+\mathrm{Na}^{+}$: 455.1836; found: 455.1831.

2-(2-((4,6-Dimethyl-5-oxo-2-thioxohexahydroimidazo[4,5-d]imidazol-1(2H)-yl)imino)methyl)phenoxy)-N-

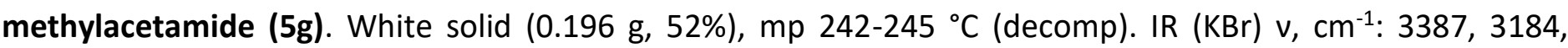
$1714,1666,1548,1500,1492,1458,1414,1402,1296,1267,1237,1213,1162,1042,749 .{ }^{1} \mathrm{H} \mathrm{NMR}(300 \mathrm{MHz}$, DMSO-d ) $\delta 2.68$ (br.s, 3H, NMe), 2.76 (s, 3H, NMe), 2.88 (s, 3H, NMe), 4.55 (d, ${ }^{2}$ J $\left.14.7 \mathrm{~Hz}, 1 \mathrm{H}, \mathrm{OCH}_{2}\right), 4.61(\mathrm{~d}$, 2J $\left.14.7 \mathrm{~Hz}, 1 \mathrm{H}, \mathrm{OCH}_{2}\right), 5.41(\mathrm{~d}, 3 \mathrm{~J} 8.0 \mathrm{~Hz}, 1 \mathrm{H}, \mathrm{CH}), 6.01(\mathrm{~d}, 3 \mathrm{~J} 8.0 \mathrm{~Hz}, 1 \mathrm{H}, \mathrm{CH}), 7.04-7.10\left(\mathrm{~m}, 2 \mathrm{H}, \mathrm{CH}_{\mathrm{arom}}\right), 7.43\left(\mathrm{t}, 3^{3} \mathrm{~J}\right.$ $\left.7.2 \mathrm{~Hz}, 1 \mathrm{H}, \mathrm{CH}_{\text {arom }}\right), 7.85-7.93\left(\mathrm{~m}, 2 \mathrm{H}, \mathrm{CH}_{\text {arom }}, \mathrm{NH}\right), 9.38(\mathrm{~s}, 1 \mathrm{H}, \mathrm{N}=\mathrm{CH}), 10.03(\mathrm{~s}, 1 \mathrm{H}, \mathrm{NH}) .{ }^{13} \mathrm{C} \mathrm{NMR}(75 \mathrm{MHz}$, 
DMSO- $\left.d_{6}\right): \delta 25.3,28.1,30.3(\mathrm{NMe}), 67.6(\mathrm{CH}), 68.1\left(\mathrm{OCH}_{2}\right), 75.1(\mathrm{CH}), 113.1\left(\mathrm{CH}_{\text {arom }}\right), 121.5\left(\mathrm{CH}_{\text {arom }}\right), 122.4$ $\left(\mathrm{C}_{\text {arom }}\right), 126.2\left(\mathrm{CH}_{\text {arom }}\right), 132.0\left(\mathrm{CH}_{\text {arom }}\right), 147.0(\mathrm{~N}=\mathrm{CH}), 156.4,157.6\left(\mathrm{C}_{\text {arom }}, \mathrm{C}=\mathrm{O}\right), 167.6(\mathrm{C}=\mathrm{O}), 178.9$ (C=S). HRMS (ESI): $m / z$ calcd for $\mathrm{C}_{16} \mathrm{H}_{20} \mathrm{~N}_{6} \mathrm{O}_{3} \mathrm{~S}+\mathrm{Na}^{+}$: 399.1210; found: 399.1202 .

\section{2-(2-(((4,6-Dimethyl-5-oxo-2-thioxohexahydroimidazo[4,5-d]imidazol-1(2H)-yl)imino)methyl)phenoxy)-N-} ethylacetamide (5h). White solid (0.222 g, 57\%), mp 216-218 ${ }^{\circ} \mathrm{C} . \mathrm{IR}(\mathrm{KBr}) \mathrm{v}, \mathrm{cm}^{-1}: 3377,3175,1741,1722$, $1657,1608,1548,1507,1490,1459,1450,1433,1401,1316,1290,1253,1235,1213,1158,1108,1073,1056$, 849, 747. ${ }^{1} \mathrm{H}$ NMR (500 MHz, DMSO-d $)^{\text {) } \delta} 1.06$ (t, $\left.{ }^{3} \mathrm{~J} 7.2 \mathrm{~Hz}, 3 \mathrm{H}, \mathrm{Me}\right), 2.76$ (s, 3H, NMe), 2.89 (s, 3H, NMe), 3.15$3.22\left(\mathrm{~m}, 2 \mathrm{H}, \mathrm{NCH}_{2}\right), 4.55$ (d, $\left.{ }^{2} \mathrm{~J} 14.4 \mathrm{~Hz}, 1 \mathrm{H}, \mathrm{OCH}_{2}\right), 4.60\left(\mathrm{~d},{ }^{2} \mathrm{~J} 14.4 \mathrm{~Hz}, 1 \mathrm{H}, \mathrm{OCH}_{2}\right), 5.41\left(\mathrm{~d},{ }^{3} \mathrm{~J} 8.2 \mathrm{~Hz}, 1 \mathrm{H}, \mathrm{CH}\right)$, 5.99 (d, 3J $8.3 \mathrm{~Hz}, 1 \mathrm{H}, \mathrm{CH}), 7.05-7.10\left(\mathrm{~m}, 2 \mathrm{H}, \mathrm{CH}_{\text {arom }}\right), 7.45\left(\mathrm{t}, 3 \mathrm{~J} 7.8 \mathrm{~Hz}, 1 \mathrm{H}, \mathrm{CH}_{\text {arom }}\right), 7.87(\mathrm{~d}, 3 \mathrm{~J} 7.7 \mathrm{~Hz}, 1 \mathrm{H}$, $\left.\mathrm{CH}_{\text {arom }}\right), 7.98\left(\mathrm{t},{ }^{3} \mathrm{~J} 5.2 \mathrm{~Hz}, 1 \mathrm{H}, \mathrm{NH}\right), 9.47(\mathrm{~s}, 1 \mathrm{H}, \mathrm{N}=\mathrm{CH}), 10.03(\mathrm{~s}, 1 \mathrm{H}, \mathrm{NH}) .{ }^{13} \mathrm{C}$ NMR $\left(125 \mathrm{MHz}, \mathrm{DMSO}-d_{6}\right): \delta 14.8$ (Me), 28.2, $30.5(\mathrm{NMe}), 33.3\left(\mathrm{NCH}_{2}\right), 67.6(\mathrm{CH}), 68.2\left(\mathrm{OCH}_{2}\right), 75.3(\mathrm{CH}), 113.2\left(\mathrm{CH}_{\text {arom }}\right), 121.6\left(\mathrm{CH}_{\text {arom }}\right), 122.5$ ( $\left.\mathrm{C}_{\text {arom }}\right), 126.1\left(\mathrm{CH}_{\text {arom }}\right), 132.1\left(\mathrm{CH}_{\text {arom }}\right), 147.0(\mathrm{~N}=\mathrm{CH}), 156.5,157.7\left(\mathrm{C}=\mathrm{O}, \mathrm{C}_{\text {arom }}\right), 166.9(\mathrm{C}=\mathrm{O}), 178.9$ (C=S). HRMS (ESI): $\mathrm{m} / z$ calcd for $\mathrm{C}_{17} \mathrm{H}_{22} \mathrm{~N}_{6} \mathrm{O}_{3} \mathrm{~S}+\mathrm{H}^{+}: 391.1547$; found: 391.1531 .

\section{Acknowledgements}

High resolution mass spectra were recorded in the Department of Structural Studies of N.D. Zelinsky Institute of Organic Chemistry, Moscow.

\section{References}

1. Mashkovskii, M. D. Lekarstvennye sredstva [Drugs]; Novaya Volna: Moscow, 2012; Vol. 1, 89.

2. Ryzhkina, I. S.; Kiseleva, Yu. V.; Murtazina, L. I.; Mishina, O. A.; Timosheva, A. P.; Sergeeva, S. Yu.; Baranov, V. V.; Kravchenko, A. N.; Konovalov A. I. Mendeleev Commun. 2015, 25, 72. https://doi.org/10.1016/j.mencom.2015.01.027

3. Yawer, M. A.; Havel, V.; Sindelar, V. Angew. Chem. Int. Ed. 2015, 54, 276. http://dx.doi.org/10.1002/anie.201409895

4. Cotelle, Y.; Hardouin-Lerouge, M.; Legoupy, S.; Alvque, O.; Levillain, E.; Hudhomme, P. Beilstein J. Org. Chem. 2015, 11, 1023. https://doi.org/10.3762/bjoc.11.115

5. Jin, X.; Hu, B. Z. Anorg. Allg. Chem. 2016, 642, 635. http://dx.doi.org/10.1002/zaac.201600100

6. Tsuchiya, S.; Cho, Y.; Konoki, K.; Nagasawa, K.; Oshima, Y.; Yotsu-Yamashita, M. Chem. Eur. J., 2015, 21, 7835.

http://dx.doi.org/10.1002/chem.201500064

7. Solel, E.; Singh, M.; Reany, O.; Keinan, E. Phys. Chem. Chem. Phys. 2016, 18, 13180. http://dx.doi.org/10.1039/c6cp00442c

8. Cow, C. N.; Harrison, P. H. M.; J. Org. Chem. 1997, 62, 8834. http://dx.doi.org/10.1021/jo9713823

9. Kam, K.; Rahimizadeh, M.; McDonald, R. S.; Harrison, P. H. M.; Chen, H.; Jenkins, S. I.; Pedrech, A. Can. J. Chem. 2005, 83, 1253. 
10. Chen, H.; Harrison, P. H. M. Can. J. Chem. 2002, 80, 601. https://doi.org/10.1139/v02-059

11. Bain, A. D.; Chen, H.; Harrison, P. H. M. Can. J. Chem. 2006, 84, 421. https://doi.org/10.1139/v06-016

12. Singh, M.; Solel, E.; Keinan, E.; Reany. O. Chem. Eur. J. 2015, 21, 536. http://dx.doi.org/10.1002/chem.201404210

13. Khaksar, S.; Vahdat, S. M.; Tajbakhsh, M.; Jahani F.; Heydari, A. Tetrahedron Lett. 2010, 51, 6388. https://doi.org/10.1016/i.tetlet.2010.09.096

14. Cao, L.; Ding, J.; Yin, G.; Gao, M.; Li, Y.; Wu, A. Synlett 2009, 1445. http://dx.doi.org/10.1055/s-0029-1216746

15. Baranov, V. V.; Gazieva, G. A.; Nelyubina, Yu. V.; Kravchenko, A. N.; Makhova, N. N. Russ. J. Org. Chem. 2011, 47, 1564 (Translation from Zh. Org. Khim. 2011, 47, 1535). http://dx.doi.org/10.1134/S1070428011100204

16. Gazieva, G. A.; Poluboyarov, P. A.; Popov, L. D.; Kolotyrkina, N. G.; Kravchenko, A. N.; Makhova, N. N. Synthesis 2012, 44, 3366. http://dx.doi.org/10.1055/s-0032-1317194

17. Gazieva, G. A.; Vikharev, Yu. B.; Anikina, L. V.; Karpova, T. B.; Kravchenko, A. N.; Permyakov, E. A.; Svitanko, I. V. Mendeleev Commun. 2013, 23, 202. https://doi.org/10.1016/j.mencom.2013.07.007

18. Gazieva, G. A.; Anikina, L. V.; Pukhov, S. A.; Karpova, T. B.; Nelyubina, Yu. V.; Kravchenko, A. N. Mol. divers. 2016, 837. http://dx.doi.org/10.1007/s11030-016-9671-1

19. Gazieva, G. A., Karpova, T. B., Nechaeva, T. V., Kravchenko A. N. Russ. Chem. Bull. 2016, 65, 2172. http://dx.doi.org/10.1007/s11172-016-1565-y

20. Jeon, A. R. ; Kim, M. E.; Park, J. K. ; Shin, W. K. ; An, D. K. Tetrahedron 2014, 70, 4420. https://doi.org/10.1016/i.tet.2014.03.045

21. Gnanaprakasam, B.; Milstein, D. J. Am. Chem. Soc. 2011, 133, 1682. http://dx.doi.org/10.1021/ja109944n

22. Han, Q.; Xiong, X.; Li, S. Catalysis Commun. 2015, $58,85$. https://doi.org/10.1016/i.catcom.2014.08.036

23. Lill, A. P.; Rodl, C. B.; Steinhilber, D.; Stark, H.; Hofmann, B. Eur. J. Med. Chem. 2015, 89, 503. https://doi.org/10.1016/i.ejmech.2014.10.054

24. Gazieva, G. A.; Vasilevskii, S. V.; Belyakov, P. A.;Nelyubina, Yu. V., Lubuzh, E. D.; Kravchenko, A. N. Mendeleev Commun. 2010, 20, 285. https://doi.org/10.1016/i.mencom.2010.09.016

25. Zhang, H.; Yu, H.; Liu, X.; Tian, L. Main Group Met. Chem. 2015, 38 (5-6), 157. https://doi.org/10.1515/mgmc-2015-0025

26. Fugard, A. J.; Thompson, B. K.; Alexandra M. Z. Slawin, A. M. Z.; Taylor, J. E.; Smith, A. D. Org. Lett. 2015, $17,5824$.

http://dx.doi.org/10.1021/acs.orglett.5b02997 